\title{
Review: HIV risk reduction interventions reduce some HIV risk behaviours in adolescents
}

Johnson BT, Carey MP, Marsh KL, et al. Interventions to reduce sexual risk for the human immunodeficiency virus in adolescents, 1985-2000: a research synthesis. Arch Pediatr Adolesc Med 2003;157:381-8.

\section{Do HIV risk reduction interventions reduce adolescent risk behaviours for HIV?}

\section{METHODS}

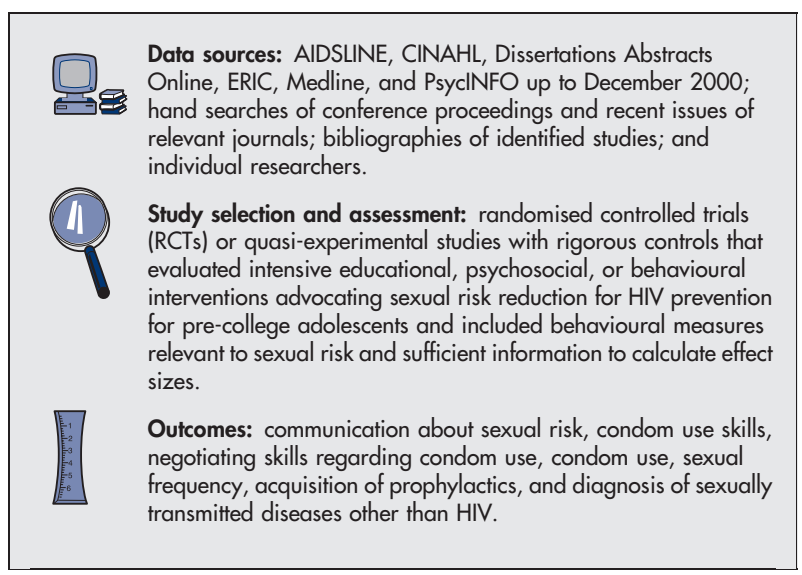

\section{MAIN RESULTS}

44 studies of 56 interventions ( $\mathrm{n}=35282$; mean age $15 \mathrm{y}$, range $11-$ 18 y; $53 \%$ African-American or African; 56\% sexually active) met the selection criteria. Meta-analysis was done using a fixed effects model. Adolescents who received HIV risk reduction interventions had improved skills in negotiating lower risk sexual encounters, increased frequency of communications with sexual partners about safer sex, reduced sexual frequency, and increased condom use compared with comparison groups (table). Risk reduction interventions did not increase acquisition of condoms or spermicide or diagnosis of sexually transmitted diseases other than HIV (table). For correspondence: $\operatorname{Dr}$ B T Johnson, Center for Health/HIV Intervention and Prevention, University of Connecticut, CT, USA. blair.t.johnson@uconn.edu Source of funding: National Institutes of Health.

\section{CONCLUSION}

HIV risk reduction interventions improve condom use skills, communication with sexual partners, and condom use and reduce sexual frequency in adolescents.

\section{Commentary}

n this review of adolescent HIV risk reduction interventions, Johnson et al used a comprehensive search strategy, explicit selection criteria, and 2 independent raters to extract study data. They obtained data directly from the researchers for about half of the 22 studies lacking sufficient information to calculate effect size estimates.

The authors used a fixed effects model for meta-analysing the data, despite significant heterogeneity in the results of studies examining condom use. They did further analyses to investigate the effect of methodological and participant characteristics on the variability of study results. Study variability was explained by several factors: condom use increased most among non-institutionalised adolescents who were given condoms and received active training in the skills needed to use and negotiate condom use with a partner. This analysis provides information of great relevance to health policy.

Public health nurses should share the findings of this systematic review to dispel the myth that HIV sexual risk reduction interventions for adolescents increase the frequency of sexual behaviour, the number of sexual partners, or the onset of sexual debut. Based on these findings, HIV prevention programmes for youth in the community should incorporate both active instruction in condom use and provision of condoms. Evaluations should focus on behavioural outcomes. Concurrently, investigators need to explore social determinants of health, including environmental structures and social processes within families, schools, and neighbourhoods[1] to identify additional factors that might promote or inhibit risk reduction behaviours in youth. Findings from such research may increase our understanding of the complex social environment that influences the adoption of risk reduction behaviours. Susan Askin, RN, PhD(c) School of Nursing, McMaster University Hamilton, Ontario, Canada

1 Roosa MW, Jones S, Tein JY, et al. Prevention science and neighborhood influences on low-income children's development: theoretical and methodological issues. Am J Community Psychol 2003;31:55-72.

HIV risk reduction interventions $v$ comparison conditions for adolescents ${ }^{*}$

\begin{tabular}{lll}
\hline Outcomes at mean 14 weeks (number of comparisons) & Mean effect size (95\% CI) & Odds ratio (CI) \\
\hline Communication with a partner (8) & $0.27(0.19$ to 0.36$)$ & $1.64(1.40$ to 1.93$)$ \\
Condom use negotiation skills (11) & $0.50(0.41$ to 0.59$)$ & $2.47(2.09$ to 2.92$)$ \\
Condom use skills (3) & $0.30(0.09$ to 0.51$)$ & $1.72(1.18$ to 2.53$)$ \\
Condom use (42) & $0.07(0.03$ to 0.11$)$ & $1.13(1.06$ to 1.21$)$ \\
Sexual frequency (38) & $0.05(0.02$ to 0.09$)$ & $1.10(1.04$ to 1.17$)$ \\
Acquired condoms or spermicide (6) & $0.13(-0.01$ to 0.26$) \dagger$ & $1.25(0.98$ to 1.60$) \dagger$ \\
Diagnosis of sexually transmitted disease other than HIV (5) & $0.03(-0.04$ to 0.11$) \dagger$ & $1.06(0.93$ to 1.22$) \dagger$ \\
\hline
\end{tabular}

*Abbreviations defined in glossary.

†Not significant. 